\title{
CONTINUITY PROPERTIES OF THE SUPERPOSITION OPERATOR
}

\author{
JÜRGEN APPELL and PJOTR P. ZABREJKO
}

(Received 4 March 1987; revised 18 November 1987)

Communicated by R. O. Vyborny

\begin{abstract}
Various continuity conditions (in norm, in measure, weakly etc.) for the nonlinear superposition operator $F x(s)=f(s, x(s))$ between spaces of measurable functions are established in terms of the generating function $f=f(s, u)$. In particular, a simple proof is given for the fact that, if $F$ is continuous in measure, then $f$ may be replaced by a function $\tilde{f}$ which generates the same superposition operator $F$ and satisfies the Carathéodory conditions. Moreover, it is shown that $F$ is weakly continuous if and only if $f$ is affine in $u$. Finally, some continuity results for the integral functional associated with the function $f$ are proved.
\end{abstract}

1980 Mathematics subject classification (Amer. Math. Soc.) (1985 Revision): 47 H 15, 46 E 30, 26 B 40,28 A 20.

Let $\Omega$ be an arbitrary set, $\mathscr{M}$ some $\sigma$-algebra of subsets of $\Omega$ (called "measurable subsets" in what follows), and $\mu$ a nonnegative countably additive and $\sigma$-finite measure on $\mathscr{M}$. Let $f=f(s, u)$ be a function defined on $\Omega \times \mathbf{R}$ and taking values in $\mathbf{R}$ (the real line). Given an arbitrary real function $x=x(s)$ on $\Omega$, by applying $f$ we get another real function $y=y(s)$ on $\Omega$ defined by $y(s)=f(s, x(s))$. In this way, the function $f$ generates a superposition operator

$$
F x(s)=f(s, x(s)) .
$$

This operator arises very often in applications, as the right-hand side of ordinary differential equations, as a mathematical model for the so-called functional link in control theory, as a generating element for some operators in the

(C) 1989 Australian Mathematical Society 0263-6115/89 \$A2.00+0.00 
theory of nonlinear oscillations, as a particularly simple example of so-called hysteresis nonlinearities, and in other fields of nonlinear analysis.

When such a problem is dealt with, one usually has to choose appropriate spaces such that the superposition operator (1) is defined and "well behaved" between these spaces. One of the most important properties of $F$ which one hardly can dispense with in applications is its continuity. One basic problem is therefore that of finding conditions on the nonlinearity $f$ (possibly both necessary and sufficient) which guarantee that the corresponding operator $F$ is continuous between the spaces under consideration. In some cases, this is very easy. For instance, the operator $F$ is obviously continuous in the space $C$ of continuous functions (over a compact domain $\Omega$ without isolated points in Euclidean space) if, and only if, the generating function $f$ is continuous on $\Omega \times \mathbf{R}$.

In many other cases it turns out, however, that the problem of characterizing continuity properties of $F$ by means of elementary properties of $f$ is surprisingly difficult. Consider, for example, the superposition operator $F$ in the space $S$ of measurable functions over $\Omega$. A classical result [5] states that the so-called Caratheodory conditions for $f$ (that is $f(s, \cdot)$ is continuous on $\mathbb{R}$ for almost all $s \in \Omega$, and $f(\cdot, u)$ is measurable on $\Omega$ for all $u \in \mathbb{R})$ are sufficient for the continuity of $F$ in the space $S$. It was an open problem for more than 40 years (called the "Nemytskij conjecture" by some people) to decide whether or not the Carathéodory conditions are also necessary. This conjecture was disproved by two famous counter-examples due to Krasnosel'skij and Pokrovskij [19], [20] and, independently, to Grande and Lipiński [10]. Nevertheless, later on it was shown by Vrkoč [46] and Ponosov [27] that a function $f$ which generates a continuous superposition operator $F$ in the space $S$, although not being necessarily a Carathéodory function, is always equivalent (in a sense to be made precise below) to a Carathéodory function. The proofs in both Vrkoč's and Ponosov's papers, however, are rather cumbersome and technical. It turns out that, by means of an appropriate modification of an approximation scheme proposed in another context by Buttazzo and DalMaso [4], one can obtain another proof which gives a clearer insight into the problem and is much simpler (and even proves a more general fact).

The purpose of the present paper is three-fold. First, we shall give a simple proof of a slightly generalized version of Vrkoč's and Ponosov's results (Theorems 1 and 2); in addition, we provide a complete characterization of continuous superposition operators between so-called ideal spaces which embrace many function spaces arising in applications (Theorem 3). Second, we shall discuss various properties of weak continuity of $F$ in ideal spaces; in particular, it turns out that if the operator $F$ is weakly continuous between two ideal function spaces, then the function $f$ must be linear in $u$ (Theorem 
4). By combining several types of convergence (strong, weak, in measure) in the domain and range of $F$, we obtain further continuity conditions for $F$ in terms of the function $f$ (Theorems 5 and 6); such results are useful, for example, in the theory of nonlinear integral equations. Third, we shall be concerned with semi-continuity properties of the integral functional

$$
\phi(x)=\int_{\Omega} f(s, x(s)) d s
$$

which is obviously closely related to the operator (1) (Theorems 7, 8 and 9).

\section{The superposition operator}

Let $\Omega, \mathscr{K}$ and $\mu$ be defined as at the beginning, and let $\lambda$ be a normalized ("probability") measure on $\mathscr{M}$ which is equivalent to $\mu$ (that is, has the same null sets). Recall (Saks' lemma) that the set $\Omega$ can be divided into two parts $\Omega_{c}$ and $\Omega_{d}$ such that $\mu$ is atomic-free ("continuous") on $\Omega_{c}$ and purely atomic ("discrete") on $\Omega_{d}$. This makes it possible to consider very general sets $\Omega$; in "natural" examples, however, one usually has $\Omega=\Omega_{c}$ (that is, one deals with "functions") or $\Omega=\Omega_{d}$ (that is, one deals with "sequences").

As usual, we denote by $S$ the set of all (equivalence classes of) almost everywhere finite measurable functions $x$ on $\Omega$. Equipped with the metric $\rho(x, y)=[x-y]$, where

$$
[z]=\inf _{0<h<\infty}\{h+\lambda(\{s|s \in \Omega,| z(s) \mid>h\})\}
$$

or

$$
[z]=\int_{\Omega} \frac{|z(s)|}{1+|z(s)|} d s
$$

the set $S$ becomes a complete metric space, and convergence with respect to this metric coincides with convergence in measure on subsets of finite measure (that is, with respect to $\lambda$ ) [7].

Besides the metric structure, the space $S$ has a natural partial ordering: $x \leq y$ means that $x(s) \leq y(s)$ a.e. on $\Omega$. Consequently, one can consider convergence with respect to this ordering [11]; this type of convergence coincides with convergence almost everywhere on $\Omega$. It is well known (Lebesgue's theorem) that convergence a.e. implies convergence in measure; the converse is true only if the measure $\mu$ is discrete (that is, $\Omega_{c}=\varnothing$ ). Nevertheless (Riesz' theorem), each sequence which is convergent in measure admist an a.e. convergent subsequence.

Given a subset $D \in \mathscr{M}$, we denote by $\chi_{D}$ the characteristic function of $D$ and by $P_{D}$ the multiplication operator

$$
P_{D} x(s)=\chi_{D}(s) x(s)
$$


Finite linear combinations of characteristic functions are usually called simple functions on $\Omega$; they form a dense subspace of $S$.

Let $f$ be a real function on $\Omega \times \mathbf{R}$, and consider the corresponding superposition operator (1). To make life easier, we shall always tacitly assume that $f(s, 0)=0$ (hence $F \theta=\theta$, where $\theta$ is the a.e. zero function); this implies, in particular, that $F P_{D}=P_{D} F$ for any $D \in \mathscr{M}$, that is, $F$ commutes with the operator (5). Of course, the condition $f(s, 0)=0$ is not really restrictive, because one can pass from the operator (1) to the operator $\tilde{F} x(s)=\tilde{f}(s, x(s))$ generated by $\tilde{f}(s, u)=f\left(s, x_{0}(s)+u\right)-f\left(s, x_{0}(s)\right)$, where $x_{0}$ is any fixed function on $\Omega$ (e.g. $x_{0}=\theta$ ).

The fact that $F$ commutes with the operator (5) implies, in particular, that $F$ maps equivalent functions into equivalent ones, that is, acts actually on classes. It is now natural to ask for acting conditions for $F$, that is, conditions on the function $f$ which ensure that the operator $F$ maps the space $S$ into itself. (If this is the case, the function $f$ is usually called superpositionally measurable [23] or sup-measurable, for short.) Surprisingly enough, this is a very hard problem; the basic difficulty in this connection lies in the fact that a sup-measurable function $f$ is by no means uniquely determined by the corresponding operator $F$, but two functions $f_{1}$ and $f_{2}$ may generate the same superposition operator and nonetheless may be "essentially different".

This gives rise to the following definition: given two functions $f_{1}$ and $f_{2}$ on a subset $\Delta \subseteq \Omega \times \mathbf{R}$, we call $f_{1}$ and $f_{2}$ superpositionally equivalent (or sup-equivalent, for short) on $\Delta$, and write

$$
f_{1}(s, u) \simeq f_{2}(s, u) \quad((s, u) \in \Delta),
$$

if the corresponding superposition operators $F_{1}$ and $F_{2}$ coincide on the set of all $x \in S$ whose graphs belong to $\Delta$. Note that sup-measurability is then invariant under the equivalence relation (6), that is, if $f$ is a sup-measurable function then so is every function $\tilde{f}$ which is sup-equivalent to $f$.

It is very striking that already functions $f$ which are sup-equivalent to the zero function $\tilde{f}(s, u) \equiv 0$ may exhibit a very pathological behaviour; such function which are nowadays called monsters in the literature (for example, in [19], [20]), will be discussed below.

Before doing so, however, we still must consider another class of functions which were introduced under the name "standard functions" in [36] and are located "between" Carathéodory functions and sup-measurable functions. A function $f$ is called a standard function if there exists a negligible set $D_{0} \in \mathscr{M}$ (that is, $D \subseteq D_{0}$ implies $D \in \mathscr{M}$ ) with the property that, for any Borel subset $C$ of $\mathbf{R}$, the set $f^{-1}(C) \backslash\left(D_{0} \times \mathbf{R}\right)$ belongs to the minimal $\sigma$-algebra which contains all products $A \times B$ with $A \in \mathscr{M}$ and $B$ a Borel subset of $\boldsymbol{R}$. For further reference, we summarize some information on this class of functions 
as a lemma. The first assertion was proved in [36], the second one in [36], [38], and the third one in [41], [42]. In particular, the proof of the last statement builds essentially on Sainte-Beuve's selection theorem [29].

Lemma 1. Every Carathéodory function and, more general, the pointwise limit of a family of Caratheodory functions is a standard function. Every standard function is sup-measurable. If $f$ is standard and the superposition operator $F$ generated by $f$ is continuous in measure, then $f$ is actually a Carathéodory function.

We are now going to consider normed spaces of measurable functions; all concepts and results presented below can be found in [49]. A Banach space $X$ of measurable functions on $\Omega$ is called ideal space if the relations $|x| \leq|y|$, $x \in S$ and $y \in X$ imply that also $x \in X$ and $\|x|X\|\leq\| y| X\|(\|x \mid X\|$ is the norm of the element $x$ in the space $X$ ). An important property of ideal spaces $X$ is that of being continuously imbedded into the space $S$. This means that every convergent sequence in $X$ is also convergent in $S$; equivalently, every ball $B_{r}(X)=\{x:\|x \mid X\| \leq r\}$ is a bounded set in $S$.

Typical examples of ideal spaces are the Lebesgue spaces $L_{p}$, defined by the norm

for $1 \leq p<\infty$, and

$$
\left\|x \mid L_{p}\right\|=\left\{\int_{\Omega}|x(s)|^{p} d s\right\}^{1 / p}
$$

$$
\left\|x\left|L_{\infty} \|=\underset{s \in \Omega}{\operatorname{ess} \sup }\right| x(s) \mid\right.
$$

for $p=\infty$, respectively, or, more generally, the Orlicz spaces $L_{M}$ defined by the (Luxemburg) norm

$$
\left\|x \mid L_{M}\right\|=\inf \left\{k \mid k>0, \int_{\Omega} M[s, x(s) / k] d s \leq 1\right\},
$$

where $M=M(s, u)$ is a Young function on $\Omega \times \mathbf{R}$ (see, for example, [21]).

Given a sup-measurable function $f$ with $f(s, 0)=0$, consider the functional

$$
\phi(x, D)=\int_{D} f(s, x(s)) d s
$$

on $S \times \mathscr{M}$. Obviously, $\phi(\theta, D)=0$ for all $D \in \mathscr{M}$, and $P_{D} x=P_{D} y$ implies that $\phi(x, D)=\phi(y, D)$. Moreover, for any $x \in S, \phi(x, \cdot)$ is a countably additive measure on the $\sigma$-algebra $\mathscr{M}$.

Given $\eta>0$, apart from (7) we define a functional $\phi_{\eta}$ by

$$
\phi_{\eta}(x, D)=\inf _{w \in S}\left\{\phi(w, D)+\eta \int_{D}|w(s)-x(s)| d s\right\} .
$$


Obviously,

$$
\phi_{\eta}(x, D) \leq \phi(x, D),
$$

and the functional $\phi_{\eta}(x, D)$ is monotonically increasing in $\eta$. The next lemma gives a relation between the functionals (7) and (8).

LEMMA 2. Let $X$ be an ideal space over some domain $\Omega$ with $\mu(\Omega)<\infty$, where the measure $\mu$ is atomic-free. Let $f$ be a sup-measurable function, and suppose that the corresponding superposition operator $F$ maps a ball $B_{r}(X)$ into the Lebesgue space $L_{1}$. Assume, moreover, that the functional (7) has the following continuity property: if a sequence $x_{n} \in B_{r}(X)$ converges in $S$ to some $x$ and is bounded by some element in $B_{r}(X)$ then

$$
\int_{D} f\left(s, x_{n}(s)\right) d s \rightarrow \int_{D} f(s, x(s)) d s
$$

as $n \rightarrow \infty$. Under these assumptions,

$$
\lim _{\eta \rightarrow \infty} \phi_{\eta}(x, D)=\phi(x, D)
$$

with $\phi_{\eta}$ defined by (8).

Proof. Let us first assume that, for almost all $s \in \Omega$, the function $f(s, \cdot)$ is constant for $|u| \geq u_{0}(s)$, where $u_{0}$ is an arbitrary (but fixed) unit in the space $X$ (see [47]). This means that we actually consider the superposition operator $F$ from the "order interval" $\left[-u_{0}, u_{0}\right]$ into the space $L_{1}$. By general boundedness results on the superposition operator (see, for example, [2, Theorem 4]), the operator $F$ is then bounded on $\left[-u_{0}, u_{0}\right]$, and hence

$$
|\phi(x, D)| \leq \int_{D}|f(s, x(s))| d s<c<\infty,
$$

where the constant $c$ depends, in general, on $D$ and $u_{0}$. But the constancy assumption on $f$ for $|u| \geq u_{0}(s)$ implies that the functional $\phi$ is bounded (by $c)$ even on the whole space $S$. In fact, given any $x \in S$, the function

$$
\tilde{x}(s)=\min \left\{|x(s)|, u_{0}(s)\right\} \operatorname{sgn} x(s)
$$

belongs to $\left[-u_{0}, u_{0}\right]$ and satisfies $|\phi(\tilde{x}, D)|=|\phi(x, D)|$. Now, by definition of the infimum (8), for $x \in\left[-u_{0}, u_{0}\right]$ we may find a $w \in S$ such that

$$
\phi(w, D)+\eta \int_{D}|w(s)-x(s)| d s \leq \phi_{\eta}(x, D)+\left(c-\phi_{\eta}(x, D)\right)=c,
$$

and hence $w \in B(x ; 2 c / \eta)$, where

$$
B(x ; \rho)=\left\{w\left|w \in S, \int_{D}\right| w(s)-x(s) \mid d s \leq \rho\right\} .
$$


Consequently,

$$
\begin{aligned}
\phi_{\eta}(x, D) & =\inf \left\{\phi(w, D)+\eta \int_{D}|w(s)-x(s)| d s \mid w \in B(x ; 2 c / \eta)\right\} \\
& \geq \inf \{\phi(w, D) \mid w \in B(x ; 2 c / \eta)\} .
\end{aligned}
$$

As $\eta \rightarrow \infty$, the "ball" $B(x ; 2 c / \eta)$ shrinks to the singleton $\{x\}$. We claim that this implies (11). In fact, if $w_{n}$ is any sequence such that

$$
\int_{D}\left|w_{n}(s)-x(s)\right| d s \rightarrow 0 \quad(n \rightarrow \infty)
$$

and $\tilde{w}_{n}$ is defined through $w_{n}$ in the same way as $\tilde{x}$ in (13) through $x$, then also

$$
\int_{D}\left|\tilde{w}_{n}(s)-\tilde{x}(s)\right| d s \rightarrow 0 \quad(n \rightarrow \infty) .
$$

Moreover, since $\tilde{w}_{n}$ belongs to $\left[-u_{0}, u_{0}\right]$ and $\left|\phi\left(\tilde{w}_{n}, D\right)\right|=\left|\phi\left(w_{n}, D\right)\right|$, we get

$$
\phi\left(w_{n}, D\right) \rightarrow \phi(x, D)(n \rightarrow \infty)
$$

as claimed. This proves the assertion in case when $f(s, \cdot)$ is constant for $|u| \geq u_{0}(s)$. If $f$ is a general function satisfying the above hypotheses, we may replace $f$ by the sequence of functions

$$
f_{n}(s, u)=f\left(s, u_{0}(s) \min \left\{u_{0}^{-1}(s)|u|, n\right\} \operatorname{sgn} u\right)
$$

and pass to the limit as $n \rightarrow \infty$.

As a consequence of Lemma 2, we get the following lemma which will be fundamental in what follows.

Lemma 3. Let $X$ be an ideal space, let $f$ be a sup-measurable function, and suppose tht the corresponding superposition operator $F$ maps a ball $B_{r}(X)$ into the Lebesgue space $L_{1}$. Assume, moreover, that the functional (7) has the continuity property indicated in Lemma 2. Then $f$ is sup-equivalent to some Carathéodory function.

Proof. Without loss of generality we may assume that $\Omega$ has finite measure (since any general $\Omega$ may be represented as a countable union of subsets $\Omega_{n}$ of finite measure), and that $\mu$ is atomic-free on $\Omega$ (since the continuity of $f(s, \cdot)$ is evident for $\left.s \in \Omega_{d}\right)$. Thus, the hypotheses of Lemma 2 are fulfilled, and the relation (11) holds. We claim that the functional $\phi_{\eta}$ satisfies a "Lipschitz-type" condition $(D \in \mathscr{M})$

$$
\left|\phi_{\eta}(x, D)-\phi_{\eta}(y, D)\right| \leq \eta \int_{D}|x(s)-y(s)| d s .
$$

In fact, if $x-y$ is integrable on $D$, we get by (8),

$$
\phi_{\eta}(x, D) \leq \phi(w, D)+\eta\left\|P_{D}(w-x) \mid L_{1}\right\| .
$$


On the other hand, given $\delta>0$, we find $w_{\delta} \in S$ such that

$$
\phi_{\eta}(y, D) \geq \phi\left(w_{\delta}, D\right)+\eta\left\|P_{D}\left(w_{\delta}-y\right) \mid L_{1}\right\|-\delta .
$$

Putting $w=w_{\delta}$ in (17) yields

$$
\begin{aligned}
\phi_{\eta}(x, D)-\phi_{\eta}(y, D) & \leq \eta\left\|P_{D}\left(w_{\delta}-x\right)\left|L_{1}\|-\eta\| P_{D}\left(w_{\delta}-y\right)\right| L_{1}\right\|+\delta \\
& \leq \eta\left\|P_{D}(x-y) \mid L_{1}\right\|+\delta .
\end{aligned}
$$

Since $\delta>0$ is arbitrary and this expression is symmetric in $x$ and $y$, we have (16). Consider now the restriction $\psi_{\eta}(\cdot, D)$ of $\phi_{\eta}(\cdot, D)$ to $\mathbf{R}$ (that is, we identify the real number $u$ with the constant function $\left.x_{u}(s) \equiv u\right)$. Condition (16) then reads

$$
\left|\psi_{\eta}(u, D)-\psi_{\eta}(v, D)\right| \leq \eta \mu(D)|u-v| .
$$

By the Radon-Nikodým theorem, the measure $\psi_{\eta}(u, \cdot)$ can be represented as an integral

$$
\psi_{\eta}(u, D)=\int_{D} g_{\eta}(s, u) d s,
$$

where the function $g_{\eta}(\cdot, u)$ is integrable over $\Omega$. Note that this function is defined only for $s \in \Omega \backslash D_{u}$, where $D_{u}$ is some null set depending, in general, on $u$. If we set

$$
D_{0}=\bigcup_{u \in \mathbf{Q}} D_{u},
$$

however, $D_{0}$ is also a null set, and the function $g_{\eta}$ extends to a function $\tilde{g}_{\eta}$ whose domain of definition $\left(\Omega \backslash D_{0}\right) \times \mathbf{R}$ does not depend any more on $u$. By the continuity of $\psi_{\eta}(\cdot, D)$ on $\mathbf{R}$ and by Lebesgue's theorem we have

$$
\psi_{\eta}(u, D)=\int_{D} \tilde{g}_{\eta}(s, u) d s,
$$

and hence

$$
\left|\int_{D}\left[\tilde{g}_{\eta}(s, u)-\tilde{g}_{\eta}(s, v)\right] d s\right| \leq \eta \mu(D)|u-v|,
$$

by (18). Since $D \in \mathscr{M}$ is arbitrary, this yields $\left|\tilde{g}_{\eta}(s, u)-\tilde{g}_{\eta}(s, v)\right| \leq \eta|u-v|$; consequently, $\tilde{g}_{\eta}$ is a Carathéodory function for each $\eta>0$. The equality (19) shows that

$$
\phi_{\eta}(x, D)=\int_{D} \tilde{g}_{\eta}(s, x(s)) d s
$$

for any constant function $x=x_{u}(u \in \mathbf{R})$. Since superposition operators commute with the multiplication operator (5), (20) holds also for all simple functions $x$, and hence just for each $x \in S$, since the simple functions are dense in $S$, and both sides of (20) are continuous functionals of $x$. 
Now let $\tilde{f}(s, u)$ be the limit of the (monotonically increasing) family $\tilde{g}_{\eta}(s, u)$ as $\eta \rightarrow \infty$. Clearly, the function $\tilde{f}$ is measurable, and by Levi's theorem we have

$$
\begin{aligned}
\phi(x, D) & =\lim _{\eta \rightarrow \infty} \phi_{\eta}(x, D)=\lim _{\eta \rightarrow \infty} \int_{D} \tilde{g}_{\eta}(s, x(s)) d s \\
& =\int_{D} \lim _{\eta \rightarrow \infty} \tilde{g}_{\eta}(s, x(s)) d s=\int_{D} \tilde{f}(s, x(s)) d s,
\end{aligned}
$$

and hence

$$
\int_{D} f(s, x(s)) d s=\int_{D} \tilde{f}(s, x(s)) d s,
$$

which means that $f(s, u) \simeq \tilde{f}(s, u)$.

It remains to show that $\tilde{f}$ is a Caratheodory function. Since all $\tilde{g}_{\eta}$ are Carathéodory functions, $\tilde{f}$ is a standard function; moreover, $\tilde{f}$ generates the continuous superposition operator $F$, and thus the assertion follows from Lemma 1.

We remark that the idea of the approximation scheme $(8)$ is contained implicitly in the paper [4].

Observe that Lemma 3 can be strengthened in the following sense: since $\phi_{\eta}(x, D) \leq \phi(x, D)$ for all $\eta>0$, only lower semicontinuity of the functional $\phi(\cdot, D)$ is required to ensure the relation $\lim _{\eta \rightarrow \infty} \phi_{\eta}(x, D)=\phi(x, D)$ in the proof of Lemma 3. In this case, $f$ becomes sup-equivalent to some function $\tilde{f}$ which is measurable in $s$ and lower semicontinuous in $u$; we shall deal with such functions in the last part (see Theorems 7, 8, and 9).

\section{Continuity in the space $S$}

As already pointed out at the beginning, the Carathéodory conditions are sufficient for the sup-measurability of a function $f$, and even for the continuity of the corresponding operator $F$ in the space $S$ (with respect to the metric (3) or (4)). The Nemytskij conjecture states that the converse is also true: if the operator $F$ is continuous in $S$, the generating function $f$ must satisfy the Carathéodory conditions. This conjecture remained open for many years and was disproved recently by two counterexamples, due to Krasnosel'skij and Pokrovskij [19], [20], and, independently, due to Grande and Lipiński [10]. Both counterexamples use the continuum hypothesis and provide constructions of extremely exotic functions $f$ on $\Omega=[0,1]$ with the following two properties: on the one hand, for any fixed $s \in[0,1]$, the function $f(s, \cdot)$ is equal to 1 for almost all $u \in \mathbf{R}$ (more precisely, for all except countably many $u$ ); on the other hand, for any fixed $x \in S$, the function $f(s, x(s))$ is equal to 0 almost everywhere on $[0,1]$ (that is, the function $f$ generates the 
zero operator)! Thus, the Nemytskij conjecture is false, since monsters generate continuous superposition operators, but certainly are not Carathéodory functions. Moreover, monsters can serve as examples of functions which are sup-measurable, but not measurable on $\Omega \times \mathbb{R}_{i}$ this answers a question raised by $Z$. Grande at the end of his monograph [9].

Now the question arises as "to what extent" the Nemytskij conjecture fails to be true; in other words, "how far" are the Caratheodory conditions for $f$ from being necessary for the continuity of $F$. In this connection, the following remarkable result was proved by Vrkoč ([46], see also [12], [13], [39], [40], [41], [42]): if a sup-measurable function $f$ generates a superposition operator $F$ which is continuous in the space $S$ (over the interval $\Omega=[0,1]$ ), then there exists a Carathéodory function $\tilde{f}$ which is sup-equivalent to $f$. For example, if $f$ is a monster, one may choose $\tilde{f}(s, u) \equiv 0$. Vrkoč's result was later generalized by Ponosov [27] to more general sets $\Omega$, and even to functions $f$ from $\Omega \times X$ into $Y$, with $X$ and $Y$ being complete metric spaces. Both Vrkoč's and Ponosov's proofs, however, are rather cumbersome. We give now a slight generalization of their results whose proof builds on Lemma 3 and is extremely simple:

THEOREM 1. Let $X$ be an ideal space, let $f$ be a sup-measurable function, and suppose that the corresponding superposition operator $F$ maps a ball $B_{r}(X)$ into the space $S$ and is continuous. Then $f$ is sup-equivalent to some Carathéodory function.

Proof. Define a function $g$ by

$$
g(s, u)=\frac{f(s, u)}{1+|f(s, u)|} .
$$

Since $F$ is continuous from $B_{r}(X)$ into $S$, the superposition operator $G$ generated by $g$ maps $B_{r}(X)$ into $L_{1}$ and satisfies the hypotheses of Lemma 3 . Consequently, $g$ is sup-equivalent to some Carathéodory function $\tilde{g}$. Since $|g(s, x(s))| \leq 1$ for all $x \in B_{r}(X)$, the same holds for $\tilde{g}$; passing, if necessary, from $\hat{g}$ to the function

$$
\tilde{\tilde{g}}(s, u)=\lim _{n \rightarrow \infty} \min \left\{\left(1-\frac{1}{n}\right),|\tilde{g}(s, u)|\right\} \operatorname{sgn} \tilde{g}(s, u),
$$

we may even assume that $|\tilde{g}(s, u)|<1$ on $\Omega \times \mathbf{R}$. Setting

$$
\tilde{f}(s, u)=\frac{\tilde{g}(s, u)}{1-|\tilde{g}(s, u)|},
$$

we obtain a Carathéodory function $\tilde{f}$ which is sup-equivalent to $f$ as claimed. 
Since continuity of $F$ in the space $S$ implies the continuity condition (10), Theorem 1 implies, in particular, the above mentioned result: every function $f$ with the property that $F$ is continuous in measure is sup-equivalent to a Carathéodory function.

It is a remarkable fact that an analogous statement holds if one restricts the operator $F$ to continuous functions: every function $f$ which generates a continuous superposition operator from the space $C$ of continuous functions into the space $S$ of measurable functions is sup-equivalent to a Caratheodory function. This follows from the fact that, by Lusin's theorem, a superposition operator in the space $S$ is completely determined by its values on the space C.

Besides convergence in measure, another important type of convergence is convergence a.e. on $\Omega$ (see the previous section). The following theorem deals with "mixed" continuity properties of the superposition operator in the space $S$ :

THEOREM 2. Let $f$ be a sup-measurable function, and suppose that the corresponding superposition operator $F$ maps every sequence which converges a.e. on $\Omega$ into a sequence which converges in measure. Then $f$ is sup-equivalent to some Carathéodory function. The same holds if $F$ maps a.e. convergent sequences into a.e. convergent sequences. On the other hand, suppose that $F$ maps every sequence which converges in measure into a sequence which converges a.e. on $\Omega$. Then the function $f(s, \cdot)$ is constant for $s \in \Omega_{c}$ and continuous for $s \in \Omega_{d}$.

Proof. The first part follows from Theorem 1, since every superposition operator which maps a.e. convergent sequences into measure-convergent sequences is continuous in $S$, and every sequence which converges with respect to the measure $\lambda$ contains an a.e. convergent subsequence.

To prove the second statement, suppose that the function $f(s, \cdot)$ is not constant for $s \in \Omega_{c}$; in this case we can find a $u \neq 0$ such that $f(s, u) \neq 0$ for $s \in D$, where $D \subseteq \Omega_{c}$ is some set of positive measure. In the usual way, considering partitions $\left\{D_{1}^{n}, D_{2}^{n}, \ldots, D_{2 n}^{n}\right\}$ of $D(n=1,2, \ldots)$ of mesh tending to zero, one obtains a sequence $\chi_{n}$ of characteristic functions which tend to zero in measure, but not a.e. on $D$. By hypothesis, the sequence $z_{n}(s)=f\left(s, u \chi_{n}(s)\right)$ tends to zero almost everywhere, contradicting the fact that $z_{n}(s)=f(s, u)$ on a subset of $D$ of positive measure. The continuity of $f(s, \cdot)$ for $s \in \Omega_{d}$ follows from the fact that convergence a.e. on $\Omega_{d}$ coincides with both pointwise convergence and convergence with respect to the measure $\lambda$. 


\section{Continuity in ideal spaces}

Given an ideal space $X$, denote by $X^{0}$ the subspace of all functions $x \in X$ with absolutely continuous norm, that is,

$$
\lim _{\lambda(D) \rightarrow 0}\left\|P_{D} x \mid X\right\|=0 .
$$

The subspace $X^{0}$ has some remarkable properties. In particular, convergent sequences in $X^{0}$ admit an easy characterization: a sequence $x_{n}$ in $X^{0}$ converges to $x \in X$ (actually, $x \in X^{0}$ ) if and only if $x_{n}$ converges to $x$ in measure and the elements $x_{n}$ have uniformly absolutely continuous norms

$$
\lim _{\lambda(D) \rightarrow 0} \sup _{n}\left\|P_{D} x_{n} \mid X\right\|=0 .
$$

Unfortunately, the subspace $X^{0}$ may be considerably "smaller" than the whole space $X$. An ideal space $X$ is called regular if $X^{0}=X$, quasi-regular if $X^{0}$ is dense in $X$ with respect to convergence in measure, and completely irregular if $X^{0}=\{\theta\}$.

For example, in the case $\Omega=\Omega_{c}$ the Lebesgue space $L_{p}$ is regular for $1 \leq p<\infty$, and completely irregular for $p=\infty$. More generally, if $X$ is the Orlicz space $L_{M}$, the space $X^{0}$ consists of all functions $x \in X$ for which

$$
\int_{\Omega} M[s, x(s) / k] d s<\infty
$$

for all $k>0$; consequently, the space $L_{M}$ is regular if and only if the Young function $M$ satisfies a $\Delta_{2}$ condition.

The aim of this section is to give continuity conditions (possibly necessary and sufficient) for the superposition operator between ideal spaces. To this end, some auxiliary notions are in order. If $X$ is an ideal space, let

$$
\delta(s)=\sup \{|x(s)||\|x \mid X\| \leq 1\},
$$

where the least upper bound is taken with respect to the partial ordering in the space $S$. In general, the function (24) may be infinite on subsets of positive measure; for instance, $\delta(s) \equiv \infty$ for $s \in \Omega_{c}$ if $X$ is quasi-regular. An illustrative example is given by the Orlicz space $L_{M}$ with Luxemburg norm (see above); in this case,

$$
\delta(s)=\sup \{u \mid M(s, u)<\infty\} .
$$

Given an open subset $G$ of $X$, let

$$
\delta(G)=\bigcup_{\left(x_{0}, r\right)}\left\{(s, u)|s \in \Omega, u \in \mathbf{R},| u-x_{0}(s) \mid \leq r \delta(s)\right\},
$$

where the union is taken over all pairs $\left(x_{0}, r\right) \in G \times(0, \infty)$ such that the ball $\left\|x-x_{0} \mid X\right\|<r$ lies in $G$. It is clear that $\delta(G)$ contains the graphs of all 
functions $x \in G$ (more precisely, the set $\{s \mid(s, x(s)) \notin \delta(G)\}$ has measure zero for each $x \in G$ ). Obviously,

$$
\delta(G)=\mathbf{\Omega} \times \mathbf{R}
$$

if $G=X$; on the other hand, equality (27) holds also for any nonempty $G \subset X$ if $X$ is quasi-regular.

It is clear from the definition that there is a close "interaction" between the behaviour of the superposition operator $F$ on a set $G \subseteq X$ and that of the generating function $f$ on the set $\delta(G) \subseteq \Omega \times \mathbf{R}$. For instance, if $G$ is the interior of the domain of definition $D(F)$ of $F$, the analytical properties of $F$ do not depend on the properties of $f$ outside $\delta(G)$.

We are now in a position to prove our basic result on the continuity of the superposition operator between ideal spaces.

THEOREM 3. Let $f$ be a sup-measurable function and suppose that the interior $G$ of the domain of definition $D(F)$ of the superposition operator $F$, considered between two ideal spaces $X$ and $Y$, is non-empty. Then, if the operator $F$ is continuous on $G$, the function $f$ is sup-equivalent to some Carathéodory function on $\delta(G)$. Conversely, if the function $f$ is sup-equivalent to some Caratheodory function on $\delta(G)$ and the space $Y$ is regular, the operator $F$ is continuous on $G$.

Proof. The first part follows from Theorem 1, since convergence in the norm of an ideal space implies convergence in measure. To prove the second part, we may assume, without loss of generality, that $f$ is a Carathéodory function on $\delta(G)$ and that $B_{r}(X) \subseteq G$ for some $r>0$; we show that $F$ is continuous at $x_{0}=\theta$.

If this is not so, we can find a sequence $x_{n}$ such that $\left\|x_{n} \mid X\right\| \leq 2^{-n} r$ and $\left\|F x_{n} \mid Y\right\| \geq \varepsilon$ for some $\varepsilon>0$. The function

$$
x_{*}=\sum_{n=1}^{\infty}\left|x_{n}\right|
$$

belongs then to $B_{r}(X)$; by the Krasnosel'skij-Ladyzhenskij lemma [18], there exists a function $z \in S$ such that $|z| \leq x_{*}$ (hence $z \in B_{r}(X)$ ) and

$$
|f(s, z(s))|=\sup \left\{|f(s, u)||| u \mid \leq x_{*}(s)\right\},
$$

(hence $|F z| \in Y$ ), because the set of all $(s, u)$ with $|u| \leq x_{*}(s)$ is contained in $\delta(G)$ and $f$ is a Carathéodory function on $\delta(G)$. Since $x_{n}$ converges in $X$ to $\theta$, $x_{n}$ converges also in measure to $\theta$, and hence, by the classical Carathéodory theorem [5], $F x_{n}$ converges in measure to $\theta$. But

$$
\lim _{\lambda(D) \rightarrow 0} \sup _{n}\left\|P_{D} F x_{n}\left|Y\left\|\leq \lim _{\lambda(D) \rightarrow 0}\right\| P_{D} F z\right| Y\right\|=0,
$$


since $|F z| \in Y$ and $Y$ is regular; consequently, $F x_{n}$ converges in $Y$ to $\theta$, a contradiction, and so we are done.

We remark that the second assertion of Theorem 3 was proved for Carathéodory functions on $\delta(G)=\Omega \times \mathbf{R}$ in [22]. Simple examples show that Theorem 3 is false without the regularity assumption on the space $Y$ [21]. Moreover, it may happen that $F$ is not continuous on the boundary of $D(F)$.

General necessary and sufficient conditions for the continuity of $F$ at some point $x_{0} \in D(F)$ can be found in [25] and [26]. These conditions imply, in particular, the following somewhat surprising result: if $F$ maps some quasiregular space $X$ into a completely irregular space $Y$ and is continuous at some point $x_{0} \in D(F)$, then $F$ is constant (that is, the function $f$ does not depend on $u$ ) [3]; in the case $X=L_{p}(1 \leq p<\infty)$ and $Y=L_{\infty}$ this is well known [50].

In special function spaces, Theorem 3 has a long history. For Lebesgue spaces, it goes back to Krasnosel'skij [16], [17] and Vajnberg [43], [44]. For Orlicz spaces, similar results can be found in [21], [28], [32], [33], [34], [35], and [45]. Finally, continuity results in abstract ideal spaces are given in [14], [15], [24], [25], [26], [37] and [48].

\section{Weak continuity in ideal spaces}

In some fields of nonlinear analysis one must consider types of convergence other than convergence in norm or in measure. In the theory of nonlinear integral equations, for example, the arising superposition operators need not be continuous in norm between two ideal spaces $X$ and $Y$, but just should map any sequence $x_{n}$ which converges in norm into a sequence which converges, say, in measure or weakly. Thus, in this and the following sections we shall provide an answer to the following question: suppose that the superposition operator $F$ acts between two ideal spaces $X$ and $Y$ and has the property that, whenever a sequence $x_{n}$ in $D(F)$ converges in a certain sense (in norm, in measure, weakly etc.) to some $x \in D(F)$, then the sequence $F x_{n}$ converges in a certain sense (the same or another) to $F x$; what can be said about the generating function $f$ ?

Recall that, given an ideal space $X$, the associate space $X^{\prime}$ is, by definition, the linear space of all functions $x \in S$ for which

$$
\langle x, y\rangle=\int_{\Omega} x(s) y(s) d s<\infty \quad(y \in X),
$$

equipped with the natural norm

$$
\left\|x \mid X^{\prime}\right\|=\sup \{\langle x, y\rangle|y \in X,\|y \mid X\| \leq 1\} .
$$


Obviously, the associate space $X^{\prime}$ is a closed (in general, strict) subspace of the usual dual space $X^{*}$ of continuous linear funtionals on $X$. One can show that $X^{\prime}=X^{*}$ if and only if $X^{0}=X$ (that is, $X$ is regular). In general, functionals $\varphi \in X^{\prime}$ can be described by special continuity properties; for instance, a functional $\varphi \in X^{*}$ belongs to $X^{\prime}$ if and only if

$$
\lim _{\lambda(D) \rightarrow 0} \sup _{|x| \leq z}\left|\varphi\left(P_{D} x\right)\right|=0
$$

for any nonnegative function $z \in X$.

It follows from the definition that $L_{\infty}=L_{1}^{\prime}\left(=L_{1}^{*}\right)$ and $L_{1}=L_{\infty}^{\prime}\left(\neq L_{\infty}^{*}\right)$. More generally, if $X$ is the Orlicz space $L_{M}$, then $X^{\prime}$ is the Orlicz spae $L_{M^{\prime}}$, where the "associate" Young function $M^{\prime}$ is defined by

$$
M^{\prime}(s, u)=\sup _{0<v<\infty}\{|u| v-M(s, v)\}
$$

(note that $M^{\prime}(s, u)=|u|^{p^{\prime}} / p^{\prime}$ if $M(s, u)=|u|^{p} / p,(1 / p)+\left(1 / p^{\prime}\right)=1$ ). Let $\Gamma$ be a total subspace of the associate space $X^{\prime}$ (see, for example, [49]). A sequence $x_{n}$ in $X$ is said to be $\Gamma$-weakly convergent to $x \in X$ if

$$
\lim _{n \rightarrow \infty}\left\langle x_{n}, z\right\rangle=\langle x, z\rangle \quad(z \in \Gamma) .
$$

For example, the relation (10) in Lemma 2 means nothing else but the $\Gamma$ weak convergence of $F x_{n}$ to $F x$ in $L_{1}$, where $\Gamma \subset L_{\infty}$ is the linear hull of all characteristic functions $\chi_{D}(D \in \mathscr{M})$. We point out that $\Gamma$-weak convergence does not imply convergence in measure, and the converse is not true either. Nevertheless, both types of convergence are compatible (in the sense that, if $x_{n}$ converges $\Gamma$-weakly to $x^{\prime}$ and in measure to $x^{\prime \prime}$, then $\left.x^{\prime}=x^{\prime \prime}\right)$.

In what follows, we shall make use of a special $\Gamma$-weakly convergent sequence of measurable functions. Let $D$ be a subset of $\Omega_{c}$ of positive (finite) measure, and construct a sequence of partitions of $D$ into sets $D\left(\varepsilon_{1}, \ldots, \varepsilon_{n}\right)$ $\left(\varepsilon_{i} \in\{0,1\}\right)$ as follows: first, let $\{D(0), D(1)\}$ be a partition of $D$ such that $\mu(D(0))=\mu(D(1))=\frac{1}{2} \mu(D)$ (recall that $D \subseteq \Omega_{c}$ ). Further, if $\left\{D\left(\varepsilon_{1}, \ldots, \varepsilon_{n}\right) \mid\right.$ $\left.\varepsilon_{i} \in\{0,1\}\right\}$ is the $n$-th partition of $D$, divide each $D\left(\varepsilon_{1}, \ldots, \varepsilon_{n}\right)$ into two parts $D\left(\varepsilon_{1}, \ldots, \varepsilon_{n}, \varepsilon_{n+1}\right)$ such that $\mu\left(D\left(\varepsilon_{1}, \ldots, \varepsilon_{n}, 0\right)\right)=\mu\left(D\left(\varepsilon_{1}, \ldots, \varepsilon_{n}, 1\right)\right)=$ $\frac{1}{2} \mu\left(D\left(\varepsilon_{1}, \ldots, \varepsilon_{n}\right)\right) ;$ this defines the $(n+1)$-st partition $\left\{D\left(\varepsilon_{1}, \ldots, \varepsilon_{n+1}\right) \mid \varepsilon_{i} \in\right.$ $\{0,1\}\}$. Now let

$$
D_{n}^{0}=\bigcup_{\varepsilon_{i} \in\{0,1\}} D\left(\varepsilon_{1}, \ldots, \varepsilon_{n}, 0\right), \quad D_{n}^{1}=\bigcup_{\varepsilon_{i} \in\{0,1\}} D\left(\varepsilon_{1}, \ldots, \varepsilon_{n}, 1\right),
$$

and observe that $\mu\left(D_{n}^{0}\right)=\mu\left(D_{n}^{1}\right)=\frac{1}{2} \mu(D)$. Obviously, the functions

$$
\theta_{n}(s)=\chi_{D_{n}^{1}}(s)-\chi_{D_{n}^{0}}(s)
$$


satisfy the orthogonality relation

$$
\int_{\Omega} \theta_{j}(s) \theta_{k}(s) d s= \begin{cases}\mu(D), & j=k, \\ 0, & j \neq k\end{cases}
$$

Consequently, we may consider Fourier series expansions with respect to the system $\left\{\theta_{n} \mid n=1,2, \ldots\right\}$ : given $x \in L_{2}(D)$, (that is, $x$ is square-integrable and vanishes outside $D$ ), we have

$$
x(s)=z(s)+\frac{1}{\mu(D)} \sum_{n=1}^{\infty}\left\{\int_{D} \theta_{n}(s) x(s) d s\right\} \theta_{n}(s),
$$

where $z$ is orthogonal to all $\theta_{n}$. Since $L_{2}(D)$ is dense in $L_{1}(D)$, and every function $x \in L_{1}$ can be written as sum $x=P_{D} x+P_{\Omega \backslash D} x$, we get

$$
\lim _{n \rightarrow \infty} \int_{\Omega} \theta_{n}(s) x(s) d s=0
$$

for all $x \in L_{1}$.

The following result will be useful in the sequel:

Lemma 4. Let $X$ be an ideal space, and suppose that $x_{1}$ and $x_{2}$ are two functions in $X$ such that the set $D=\left\{s: x_{1}(s) \neq x_{2}(s)\right\}$ is contained in $\Omega_{c}$ and has finite measure. Then the sequence

$$
z_{n}(s)=\frac{1+\theta_{n}(s)}{2} x_{1}(s)+\frac{1-\theta_{n}(s)}{2} x_{2}(s)
$$

converges $X^{\prime}$-weakly to the function

$$
z(s)=\frac{x_{1}(s)+x_{2}(s)}{2} .
$$

Proof. The assertion is almost obvious: given $y \in X^{\prime}$, we have $x_{1} y \in L_{1}$, $x_{2} y \in L_{1}$, and

$$
\lim _{n \rightarrow \infty}\left\langle z_{n}, y\right\rangle=\left\langle\frac{x_{1}+x_{2}}{2}, y\right\rangle+\lim _{n \rightarrow \infty}\left\langle\theta_{n}, \frac{x_{1}-x_{2}}{2} y\right\rangle=\langle z, y\rangle .
$$

Let $X$ and $Y$ be two ideal spaces. Recall [47] that the set $Y / X$ consists of all functions $z \in S$ such that $x z \in Y$ for each $x \in X$; equipped with the natural norm

$$
\|z \mid Y / X\|=\sup \{\|x z|Y\|:\| x| X\| \leq 1\},
$$

the set $Y / X$ becomes an ideal space, the multiplicator space of $X$ and $Y$ (observe that $X^{\prime}=L_{1} / X$, as can be seen by a comparison of (28) and (29)).

For example, if $X=L_{M}$ and $Y=L_{N}$ are two Orlicz spaces generated by the Young functions $M=M(u)$ and $N=N(u)$, respectively, the space $Y / X$ contains only the zero function if $L_{M}$ is not imbedded into $L_{N}$, coincides 
with $L_{\infty}$ if $L_{M}$ is imbedded into $L_{N}$, but the imbedding is not absolutely continuous, and is isomorphic to the Orlicz space $L_{R}$ generated by the Young function

$$
R(u)=\sup _{0<v<\infty}\{N(|u| v)-M(v)\}
$$

if $L_{M}$ is absolutely continuously imbedded into $L_{N}$ (see [1], [47]). In particular, if $X=L_{p}$ and $Y=L_{q}$ are two Lebesgue spaces (that is, $M(u)=|u|^{p} / p$ and $\left.N(u)=|u|^{q} / q\right)$, the three cases mentioned above correspond to the cases $p<q, p=q$ and $p>q$, and $R(u)=|u|^{r} / r$ with $1 / r=(1 / q)-(1 / p)$ in the third case.

We are now ready to formulate a condition (both necessary and sufficient) for the $(\Gamma, \Delta)$-weak continuity of $F$ between two ideal spaces $X$ and $Y$ (which means that $\Gamma$ and $\Delta$ are total subspaces of $X^{\prime}$ and $Y^{\prime}$, respectively, and $F$ maps $\Gamma$-weakly convergent sequences into $\Delta$-weakly convergent sequences.)

Theorem 4. Let $X$ and $Y$ be two ideal spaces and let $\Gamma$ and $\Delta$ be total subspaces of $X^{\prime}$ and $Y^{\prime}$, respectively. Let $f$ be a sup-measurable function, and denote by $D(F)$ the domain of definition of the superposition operator $F$, considered between $X$ and $Y$. Then $F$ is $(\Gamma, \Delta)$-weakly continuous if and only if the following two conditions hold:

(a) the restriction of $f$ to $\Omega_{c} \times \mathbf{R}$ satisfies

$$
f(s, u) \simeq a(s)+b(s) u \quad\left((s, u) \in \delta(D(F)) \cap\left(\Omega_{c} \times \mathbf{R}\right)\right),
$$

where $a \in Y$ and $b \in(Y / X) \cap(\Gamma / \Delta)$;

(b) the restriction of $f$ to $\Omega_{d} \times \mathbf{R}$ is a Caratheodory function.

Proof. Without loss of generality, we can treat the two cases $\Omega=\Omega_{c}$ and $\Omega=\Omega_{d}$ separately.

Let first $\Omega=\Omega_{c}$. If $f$ has the form (30) and $x_{n}$ converges $\Gamma$-weakly to $x$, then for any $y \in \Delta$

$$
\lim _{n \rightarrow \infty}\left\langle F x_{n}-F x, y\right\rangle=\lim _{n \rightarrow \infty}\left\langle b\left(x_{n}-x\right), y\right\rangle=\lim _{n \rightarrow \infty}\left\langle x_{n}-x, b y\right\rangle=0,
$$

and hence $F x_{n}$ converges $\Delta$-weakly to $F x$.

The proof of the necessity of (a) is somewhat harder. First, we claim that the function $f$ is sup-equivalent to an affine function on $\delta(D(F))$. If this is not so, we can find two functions $x_{1}, x_{2} \in D(F)$ such that

$$
f\left(s, \frac{1}{2}\left[x_{1}(s)+x_{2}(s)\right]\right)-\frac{1}{2}\left[f\left(s, x_{1}(s)\right)+f\left(s, x_{2}(s)\right)\right] \neq 0 \quad(s \in D)
$$

on a set $D \subset \Omega_{c}$ of positive measure. By Lemma 4 , the sequence

$$
z_{n}(s)=\frac{1+\theta_{n}(s)}{2} x_{1}(s)+\frac{1-\theta_{n}(s)}{2} x_{2}(s)
$$


converges $\Gamma$-weakly to the function $z=\frac{1}{2}\left[x_{1}+x_{2}\right]$. By hypothesis, $F$ is $(\Gamma, \Delta)$-weakly continuous, and thus the sequence $F z_{n}$ converges $\Delta$-weakly to the function $F z=F\left(\frac{1}{2}\left[x_{1}+x_{2}\right]\right)$. On the other hand, again by Lemma 4, $F x_{n}$ converges $\Delta$-weakly to the function $\frac{1}{2}\left[F x_{1}+F x_{2}\right]$, contradicting (31). Consequently, the function $f$ is affine on $\delta(D(F))$. Moreover, the acting condition $F(D(F)) \subseteq Y$ and the $(\Gamma, \Delta)$-weak continuity of $F$ imply that $a \in Y$ and $b \in(Y / X) \cap(\Gamma / \Delta)$.

It remains to show that $f$ is a Caratheodory function on $\Omega_{d} \times \mathbf{R}$. But this is obvious, since $\Gamma$ weak convergence implies pointwise convergence.

In the case of a Caratheodory function $f$, Theorem 4 was proved by Shragin for Lebesgue spaces in [30] and for Orlicz spaces in [31]. Moreover, the first part of Theorem 4 is mentioned (without proof) at the end of [31] in the case when $\Omega$ is a bounded domain in Euclidean space (with Lebesgue measure), $f$ is a Carathéodory function, and $X$ is regular. Obviously, all these assumptions are not necessary.

\section{Other continuity properties}

Now we shall deal with "mixed" continuity properties of the superposition operator between ideal spaces. To be specific, we shall consider the operator $F$ between two ideal spaces $X$ and $Y$, and suppose that $F$ maps any sequence in $B_{r}(X)$ which converges in measure into a sequence in $Y$ which converges $Y^{\prime}$-weakly, or, vice versa, that $F$ maps any sequence in $B_{r}(X)$ which converges $X^{\prime}$-weakly into a sequence in $Y$ which converges in measure; in the first case $F$ will be called $\left(S, Y^{\prime}\right)$-continuous, in the second case $\left(X^{\prime}, S\right)$-continuous.

At first glance, such continuity concepts may seem to be somewhat artificial, but they arise rather often in the theory of integral equations and in variational methods of nonlinear analysis (see the remark after Theorem 5).

Theorem 5. Let $X$ and $Y$ be two ideal spaces, and suppose that $f$ is a sup-measurable function which generates an $\left(S, Y^{\prime}\right)$-continuous superposition operator $F$ from $B_{r}(X)$ into $Y$. Then $f$ is sup-equivalent to some Carathéodory function. Moreover, for each $y \in Y^{\prime}$ one has

$$
\lim _{\lambda(D) \rightarrow 0} \sup _{x \in B_{r}(X)}\left\langle P_{D} F x, y\right\rangle=0 .
$$

Proof. Suppose first that $Y=L_{1}$. If $x_{n}$ is a sequence in $B_{r}(X)$ which converges in measure to $x$, the sequence $F x_{n}$ converges, by assumption, $L_{\infty}$ weakly to $F x$; in particular, $\phi\left(F x_{n}, D\right)=\left\langle F x_{n}, \chi_{D}\right\rangle$ converges to $\phi(F x, D)=$ $\left\langle F x, \chi_{D}\right\rangle$ for any $D \in \mathscr{M}$ (see (10)). By Lemma $3, f$ is sup-equivalent to some 
Carathéodory function. Moreover, by the Hahn-Saks-Vitali theorem [7], we have

$$
\lim _{\lambda(D) \rightarrow 0} \sup _{n}\left|\int_{D} F x_{n}(s) d s\right|=0
$$

this shows that (32) holds, and thus the assertion is proved in case $Y=L_{1}$.

Now, if $Y$ is an arbitrary ideal space, $F$ is $\left(S, Y^{\prime}\right)$-continuous from $B_{r}(X)$ into $Y$, and $y \in Y^{\prime}$ is a unit in $Y^{\prime}$ (see [49]), the superposition operator $F_{y}$ generated by $f_{y}(s, u)=f(s, u) y(s)$ is $\left(S, L_{\infty}\right)$-continuous from $B_{r}(X)$ into $L_{1}$. By what has been proved above, there exists a Carathéodory function $\tilde{f}_{y}$ which is sup-equivalent to $f_{y}$, and hence $\tilde{f}(s, u)=\tilde{f}_{u}(s, u) y(s)^{-1}$ is a Carathéodory function which is sup-equivalent to $f$. The assertion (32) follows from the definition of $f_{y}$.

A comparison of Theorem 1 and Theorem 5 shows that the hypothesis of $\left(S, Y^{\prime}\right)$-continuity is reflected in the additional property (32). We point out that (32) in the case $Y=L_{1}$ is equivalent to the absolute boundedness of $F\left(B_{r}(X)\right)$, that is, the elements of $F\left(B_{r}(X)\right)$ have uniformly absolutely continuous norms (cf. (23)):

$$
\lim _{\lambda(D) \rightarrow 0} \sup _{x \in B_{r}(X)}\left\|P_{D} F x \mid L_{1}\right\|=0 .
$$

A classical result ([6], see also [7]) states that this in turn is equivalent to the weak compactness of $F\left(B_{r}(X)\right)$ in $L_{1}$.

The following example shows how (32) may be useful in applications. Suppose, for instance, that

$$
K x(s)=\int_{\Omega} k(s, t) x(t) d t
$$

is a linear integral operator between $L_{1}$ and $L_{p}$ with the property that the operator

$$
|K| x(s)=\int_{\Omega}|k(s, t)| x(t) d t
$$

also maps $L_{1}$ into $L_{p}$. Then $K$ maps every absolutely bounded subset of $L_{1}$ into a relatively compact subset of $L_{p}$ (see [47], [51]). On the other hand, if (32) holds for $X=L_{p}$ and $Y=L_{1}$, then $F$ maps every bounded subset of $L_{p}$ into an absolutely bounded subset of $L_{1}$ (in [51] such operators are called "improving"). Thus, the Schauder fuxed point principle applies to the Hammerstein integral equation $x=K F x$ in $L_{p}$, although, in general, neither of the "factors" $K$ or $F$ is compact.

To conclude, we present a result analogous to Theorem 5 , where only the sense of continuity is "reversed". It shows that there are "very few" $\left(X^{\prime}, S\right)$ continuous operators; the proof is very similar to that of Theorem 3 , and therefore we shall not present it. 
Theorem 6. Let $X$ and $Y$ be two ideal spaces, and suppose that $f$ is a sup-measurable function which generates an $\left(X^{\prime}, S\right)$-continuous superposition operator $F$ from $B_{r}(X)$ into $Y$. Then the function $f(s, \cdot)$ is constant for $s \in \Omega_{c}$ and continuous for $s \in \boldsymbol{\Omega}_{d}$.

\section{Continuity properties of integral functionals}

In this section we shall discuss some continuity properties of the integral functional

$$
\phi(x)=\int_{\Omega} f(s, x(s)) d s
$$

generated by some sup-measurable function $f$. Obviously, this functional is defined on a subset of some ideal space $X$ if the corresponding superposition operator

$$
F x(s)=f(s, x(s))
$$

maps this subset into the space $L_{1}$. Our aim is therefore to find relations between the continuity properties of the functional (33) and the operator (34).

Much general information on such integral functionals can be found, for example, in the monograph [8]. We begin with a general continuity result.

LEMMA 5. The functional (33) is continuous in the norm of $X$ (in measure, $\Gamma$-weakly, respectively) on the interior $G$ of its domain of definition in $X$ if and only if the corresponding superposition operator (34) maps any bounded sequence in $X$ which converges in norm (in measure, $\Gamma$-weakly, respectively) into a $L_{\infty}$-weakly convergent sequence in $L_{1}$.

Proof. Since the "if" part is trivial, we shall prove only the "only if" part. All three statements are proved similarly; therefore we shall restrict ourselves to the case when $\phi$ is, say, $\Gamma$-weakly continuous.

Thus, suppose that $x_{n}$ is a bounded sequence (without loss of generality, $\left.\left\|x_{n} \mid X\right\| \leq 1\right)$ in $X$ which converges $\Gamma$-weakly to $x \in X$. Since $\left\langle P_{D} x, y\right\rangle=$ $\left\langle x, P_{D} y\right\rangle$, every sequence $P_{D} x_{n}$ converges $\Gamma$-weakly to $P_{D} x$, and hence

$$
\lim _{n \rightarrow \infty} \int_{D} f\left(s, x_{n}(s)\right) d x=\int_{D} f(s, x(s)) d s .
$$

By the Hahn-Saks-Vitali theorem, this means that the sequence $F x_{n}$ converges $L_{\infty}$-weakly to $F x$ in $L_{1}$. 
When dealing with functionals like (33) in applications, it often suffices to prove the lower semi-continuity of $\phi$, that is, the convergence of $x_{n}$ to $x$ (in some sense) implies that

$$
\phi(x) \leq \varliminf_{n \rightarrow \infty} \phi\left(x_{n}\right) .
$$

The methods developed above allow us to study also semicontinuity properties of this functionals. The corresponding results will be given in the following three theorems. Suppose first that (35) holds if $x_{n}$ converges to $x$ in norm. In this case, with the same reasoning as in Lemma 3, we get that the function $\tilde{f}$ constructed there is lower semi-continuous in $u$. For shortness, we call $f$ a lower semi-Carathéodory function if $f(s, \cdot)$ is lower semi-continuous on $\mathbf{R}$ for almost all $s \in \Omega$, and $f(\cdot, u)$ is measurable on $\Omega$ for all $u \in \mathbf{R}$. Thus we have

THEOREM 7. Let $X$ be an ideal space, and suppose that the domain of definition $D(\phi)$ of the functional $\phi$ has non-empty interior. Then $\phi$ satisfies (35) for any bounded sequence $x_{n}$ in $X$ which converges in norm to $x$ if and only if $\phi$ is generated by some lower semi-Carathéodory function $f$.

Some more must be required if we consider convergence in measure.

THEOREM 8. Let $X$ be an ideal space, and suppose that the domain of definition $D(\phi)$ of the functional $\phi$ has non-empty interior. Then $\phi$ satisfies (35) for any bounded sequence $x_{n}$ in $D(\phi)$ which converges in measure to $x$ if and only if $\phi$ is generated by some lower semi-Carathéodory function $f$, and the superposition operator $F_{-}$generated by the function

$$
f_{-}(s, u)=\min \{f(s, u), 0\}
$$

maps bounded subsets of $X$ into absolutely bounded subsets of $L_{1}$.

Proof. The "if" part follows from the "Fatou-property" of the integral. To prove the "only if" part, let $x_{n}$ be a bounded sequence in $D(\phi)$ which converges in measure to $x$. Let

$$
\tilde{x}_{n}(s)= \begin{cases}x_{n}(s) & \text { if } f\left(s, x_{n}(s)\right)<0, \\ 0 & \text { if } f\left(s, x_{n}(s)\right) \geq 0,\end{cases}
$$

and define $\tilde{x}$ through $x$ analogously. The sequence $\tilde{x}_{n}$ is also bounded in norm and converges in measure to $\tilde{x}$; consequently, $\phi\left(\tilde{x}_{n}\right)$ converges to $\phi(\tilde{x})$. But

$$
\phi\left(\tilde{x}_{n}\right)=\phi_{-}\left(x_{n}\right), \quad \phi(\tilde{x})=\phi_{-}(x)
$$


where

$$
\phi_{-}(x)=\int_{\Omega} f_{-}(s, x(s)) d s .
$$

This shows that $\phi_{-}$is also lower semi-continuous (with respect to convergence in measure). On the other hand, by Fatou's lemma $\phi_{-}$is upper semicontinuous, and hence continuous in $X$. The statement follows now from Lemma 5.

It remains to analyze the lower semi-continuity of the functional (33) with respect to $\Gamma$-weak convergence; here we shall restrict ourselves to $\Gamma=X^{\prime}$.

THEOREM 9. Let $X$ be an ideal space, and suppose that the domain of definition $D(\phi)$ of the functional $\phi$ has non-empty interior $G$. Then $\phi$ satisfies (35) for any bounded sequence $x_{n}$ in $X$ which converges $X^{\prime}$-weakly to $x$ if and only if $\phi$ is generated by some lower semi-Carathéodory function $f$ which is convex on $\delta(G)$ with respect to $u$, and the superposition operator $\hat{F}$ generated by the derivative

$$
\hat{f}(s, u)=f_{u}^{\prime}(s, u)
$$

maps $G$ into $X^{\prime}$.

Proof. To prove the "if" part, let $x_{n}$ be a bounded sequence in $X$ which converges $X^{\prime}$-weakly to $x$. By the convexity of $f(s, \cdot)$,

$$
F x_{n}(s)-F x(s) \geq \hat{F} x(s)\left[x_{n}(s)-x(s)\right],
$$

and hence

$$
\varliminf_{n \rightarrow \infty} \phi\left(x_{n}\right)-\phi(x) \geq \lim _{n \rightarrow \infty}\left\langle\hat{F} x, x_{n}-x\right\rangle=0
$$

since $\hat{F} x \in X^{\prime}$.

To prove the "only if" part, we show first that the function $f(s, \cdot)$ is convex. If this is not so, we can find two functions $x_{1}, x_{2} \in X$ whose graphs lie in $\delta(G)$ and which satisfy

$$
f\left(s, \frac{1}{2}\left[x_{1}(s)+x_{2}(s)\right]\right)>\frac{1}{2}\left[f\left(s, x_{1}(s)\right)+f\left(s, x_{2}(s)\right)\right] \quad(s \in D)
$$

on a set $D \subseteq \Omega$ of positive measure (outside $D$ we may put $x_{1}=x_{2}=\theta$ ). The sequence $z_{n}$ constructed in Lemma 4 converges $X^{\prime}$-weakly to $z=\frac{1}{2}\left[x_{1}+x_{2}\right]$; by assumption,

$$
\phi\left(\frac{1}{2}\left[x_{1}+x_{2}\right]\right) \leq \lim _{n \rightarrow \infty} \phi\left(z_{n}\right) .
$$

Again by Lemma $4, \phi\left(z_{n}\right)$ converges to $\frac{1}{2}\left[\phi\left(x_{1}\right)+\phi\left(x_{2}\right)\right]$, contradicting (36). This shows that $f(s, \cdot)$ is a convex function.

It remains to prove that $\hat{F}$ maps $G$ into $X^{\prime}$. Let $x \in G$ and $h \in X$ be such that $x+h$ belongs to the domain of definition of $\phi$. The inequality

$$
\hat{f}(s, x(s)) h(s) \leq f(s, x(s)+h(s))-f(s, x(s)),
$$


implies that

$$
\langle\hat{F} x, h\rangle \leq \phi(x+h)-\phi(x)<\infty,
$$

hence $\hat{F} x \in X^{\prime}$ as claimed.

\section{Acknowledgement}

The authors express their gratitude to both the known and unknown referee for their very valuable remarks and improvements.

\section{References}

[1] T. Andô, 'On products of Orlicz spaces', Math. Ann. 140 (1960), 174-186.

[2] J. Appell and P. P. Zabrejko, 'Boundedness properties of the superposition operator,' Bull. Polish Acad. Sci. (to appear).

[3] V. A. Bondarenko, 'Cases of degeneracy of the superposition operator in function spaces' (Russian), Jaroslav. Gos. Univ. Kach. Priblizh. Metody Issled. Oper. Uravn. 1 (1976), 11-16.

[4] G. Buttazzo and G. Dal Maso, 'On Nemyckii operators and integral representations of local functionals,' Rend. Mat. 3 (1983), 491-509.

[5] K. Carathéodory, Vorlesungen über reelle Funktionen, (De Gruyter, Leipzig-Berlin, 1918).

[6] N. Dunford and B. J. Pettis, 'Linear operators on summable functions,' Trans. Amer. Math. Soc. 47 (1940), 323-392.

[7] N. Dunford and J. Schwartz, Linear operators. 1, (Interscience, Leyden, 1963).

[8] E. Giner, Etudes sur les fonctionelles intégrales, (Doctoral Disssertation, Univ. Pau, 1985).

[9] Z. Grande, 'La measurabilité des fonctions de deux variables et de la superposition $F(x, f(x))$,' Dissertationes Math. Warszawa 159 (1979), 1-45.

[10] Z. Grande and J. S. Lipiński, 'Un exemple d'une fonction sup-mesurable qui n'est pas mesurable,' Colloq. Math. 39 (1978), 77-79.

[11] L. V. Kantorovich, B. Z. Vulih and A. G. Pinsker, Functional analysis in semi-ordered spaces (Russian), Gostehizdat, Moskva, 1950.

[12] K. Karták, 'A generalization of the Carathéodory theory of differential equations,' Czechoslovak Math. J. 17 (1967), 482-514.

[13] K. Karták, 'On Carathéodory operators,' Czechoslovak Math. J. 17 (1967), 515-519.

[14] N. V. Kirpotina, 'On the continuity of the Nemytskij operator in Banach spaces' (Russian), Metody Reor. Diff. Uravn. Prilozh. Moskva 339 (1975), 76-81.

[15] W. Kozłowski, 'Nonlinear operators in Banach function spaces,' Comm. Math. Prace Mat. 22 (1980), 85-103.

[16] M. A. Krasnosel'skij, 'On the continuity of the operator $F u(x)=f(x, u(x))$ ' (Russian), Dokl. Akad. Nauk SSSR 77 (1951), 185-188.

[17] M. A. Krasnosel'skij, Topological methods in the theory of nonlinear integral equations (Russian), Gostehizdat, Moskva, 1956 [English transl.: Macmillan, New York, 1964].

[18] M. A. Krasnosel'skij and L. A. Ladyzhenskij, 'Conditions for the complete continuity of the P. S. Uryson operator' (Russian), Trudy Moskov. Mat. Obshch. 3 (1954), 307-320.

[19] M. A. Krasnosel'skij and A. V. Pokrovskij, 'On a discontinuous superposition operator' (Russian), Uspehi Mat. Nauk 32 (1977), 169-170. 
[20] M. A. Krasnosel'skij and A. V. Pokrovskij, 'Equations with discontinuous nonlinearities' (Russian), Dokl. Akad. Nauk SSSR 248 (1979), 1056-1059 [= Soviet Math. Dokl. 20 (1979), 1117-1120].

[21] M. A. Krasnosel'skij and Ja. B. Rutitskij, Convex functions and Orlicz spaces (Russian), Fizmatgiz, Moskva, 1958 [English transl.: Noordhoff, Groningen, 1961].

[22] M. A. Krasnosel'skij, Ja. B. Rutitskij and R. M. Sultanov, 'On a nonlinear operator which acts in a space of abstract functions' (Russian), Izv. Akad. Nauk Azerbajdzh. SSR, Ser. Fiz.-Teh. Mat. Nauk 3 (1959), 15-21.

[23] M. A. Krasnosel'skij, P. P. Zabrejko, Je. I. Pustyl'nik and P. Je. Sobolevskij, Integral operators in spaces of summable functions (Russian), Nauka, Moskva, 1966 [English transl.: Noordhoff, Leyden, 1976].

[24] P. Mänz, 'On the continuity of the superposition operator acting in normed product spaces' (Russian), Litovsk. Mat. Sb. 7 (1967), 289-296.

[25] A. S. Makarov, 'Continuity criteria for the superposition operator' (Russian), Prilozh. Funk. Anal. Priblizh. Vychisl. Kazan. Univ. (1974), 80-83.

[26] P. M. Obradovich, 'On the continuity of the superposition operator' (Russian), Voronezh. Gos. Univ. Probl. Mat. Anal. Slozhn. Sistem 2 (1968), 78-80.

[27] A. V. Ponosov, 'On the Nemytskij conjecture' (Russian), Dokl. Akad. Nauk SSSR 289 (1986), 1308-1311. [Soviet Math. Dokl. 34 (1987), 231-233.]

[28] Ja. B. Rutitskij, 'On a nonlinear operator in Orlicz spaces' (Ukrainian), Dopovidi Akad. Nauk Ukr. SSR 3 (1952), 161-163.

[29] M. F. Sainte-Beuve, 'On the extension of von Neumann-Aumann's theorem', J. Funct. Anal. 17 (1974), 112-129.

[30] I. V. Shragin, 'On the weak continuity of the Nemytskij operator' (Russian), Uchen. Zapiski Moskov. Obl. Ped. Inst. 57 (1957), 73-79.

[31] I. V. Shragin, 'On the weak continuity of the Nemytskij operator in generalized Orlicz spaces' (Russian), Uchen. Zapiski Moskov. Obl. Ped. Inst. 77 (1959), 169-179.

[32] I. V. Shragin, 'On the continuity of the Nemytskij operator in Orlicz spaces' (Russian), Uchen. Zapiski Moskov. Obl. Ped. Inst. 70 (1959), 49-51.

[33] I. V. Shragin, 'On the continuity of the Nemytskij operator in Orlicz spaces' (Russian), Dokl. Akad. Nauk SSSR 140, 3 (1961), 543-545 [= Soviet Math. Dokl. 2 (1961), 12461248].

[34] I. V. Shragin, On the continuity of the Nemytskij operator (Russian), Trudy 5- $j$ Vsjesojuznoj Konf. Funk. Anal. Akad. Nauk Azerbajdzh. SSR Baku (1961), 272-277.

[35] I. V. Shragin, 'On the continuity of the Nemytskij operator in Orlicz spaces' (Russian), Kishin. Gos. Univ. Zapiski 70 (1964), 49-51.

[36] I. V. Shragin, 'Conditions for the measurability of superpositions' (Russian), Dokl. Akad. Nauk SSSR 197 (1971), 295-298 [= Soviet Math., Dokl. 12 (1971) 465-470], Erratum: Dokl. Akad. Nauk SSSR 200 (1971), vii.

[37] I. V. Shragin, 'The superposition operator in modular function spaces' (Russian), Studia Math. 43 (1972), 61-75.

[38] I. V. Shragin, 'Superpositional measurability' (Russian), Izv. Vyssh. Uchebn. Zaved. Mat. 1 (1975), 82-89.

[39] I. V. Shragin, 'Abstract Nemytskij operators are locally defined operators' (Russian), Dokl. Akad. Nauk SSSR 227 (1976), 47-49 [= Soviet Math. Dokl. 17 (1976), 354-357].

[40] I. V. Shragin, 'On the continuity of locally defined operators' (Russian), Dokl. Akad. Nauk SSSR 232 (1977), 292-295 [= Soviet Math. Dokl. 18 (1977), 75-78].

[41] I. V. Shragin, 'The necessity of the Caratheodory conditions for the continuity of the Nemytskij operator' (Russian), Perm': Gos. Polit. Inst., Funkc.-Diff. Krajev. Zad. Mat. Fiz. (1978), 128-134.

[42] I. V. Shragin, 'On the Carathéodory conditions' (Russian), Uspehi Mat. Nauk 34 (1979), 219-220 [= Russian Math. Surveys 34 (1970), 220-221]. 
[43] M. M. Vajnberg, 'On the continuity of some special operators' (Russian), Dokl. Akad. Nauk SSSR 73 (1950), 253-255.

[44] M. M. Vajnberg, Variational methods in the study of nonlinear operators (Russian), Gostehizdat, Moskva, 1956 [English transl.: Holden-Day, San Francisco-London-Amsterdam, 1964].

[45] M. M. Vajnberg and I. V. Shragin, The Nemytskij operator in generalized Orlicz spaces (Russian), Uchen. Zapiski Moskov. Obl. Ped. Inst. 77 (1959), 145-159.

[46] I. Vrkoč, 'The representation of Carathéodory operators', Czechoslovak Math. J. 19 (1969), 99-109.

[47] P. P. Zabrejko. 'Nonlinear integral operators' (Russian), Voronezh. Gos. Univ. Trudy Sem. Funk. Anal. 8 (1966), 1-148.

[48] P. P. Zabrejko, On the theory of integral operators in ideal function spaces (Russian), (Doctoral Dissertation, Univ. Voronezh, 1968).

[49] P. P. Zabrejko, 'Ideal function spaces. I' (Russian), Jaroslav. Gos. Univ. Vestnik 8 (1974), 12-52.

[50] P. P. Zabrejko, A. I. Koshelev, M. A. Krasnosel'skij, S. G. Mihlin, L. S. Rakovshchik and V. Ja. Stetsenko, Integral equations (Russian), Nauka, Moskva, 1968 [English transl.: Noordhoff, Leyden, 1975].

[51] P. P. Zabrejko and Je. I. Pustyl' nik, 'On the continuity and complete continuity of nonlinear integral operators in $L_{p}$ spaces' (Russian), Uspehi Mat. Nauk 19 (1964), 204-205.

Institut für Mathematik

Universität Würzburg

Am Hubland

D-8700 Würzburg

West Germany
Mech.-Mat. Fakultet Belgosuniversitet Minsk

Pl. Lenina 1 SU-220080 Minsk

USSR 